\title{
Analysis on the Levi-Civita field, a brief overview
}

\author{
Khodr Shamseddine and Martin Berz
}

\begin{abstract}
In this paper, we review the algebraic properties of various nonArchimedean ordered structures, extending them in various steps which lead naturally to the smallest non-Archimedean ordered field that is Cauchy-complete and real closed. In fact, the Levi-Civita field is small enough to allow for the calculus on the field to be implemented on a computer and used in applications such as the fast and accurate computation of the derivatives of real functions as "differential quotients" up to very high orders.

We then give an overview of recent research on the Levi-Civita field. In particular, we summarize the convergence and analytical properties of power series, showing that they have the same smoothness behavior as real power series; and we present a Lebesgue-like measure and integration theory on the field. Moreover, based on continuity and differentiability concepts that are stronger than the topological ones, we discuss solutions to one-dimensional and multi-dimensional optimization problems as well as existence and uniqueness of solutions of ordinary differential equations.
\end{abstract}

\section{Introduction}

The real numbers owe their fundamental role in Mathematics and the sciences to certain special properties. To begin, like all fields, they allow arithmetic calculation. Furthermore, they allow measurement; any result of even the finest measurement can be expressed as a real number. Additionally, they allow expression of geometric concepts, which requires the existence of roots, a property that at the same time is beneficial for algebra. Moreover, they allow the introduction of certain transcendental functions such as the exponential function, which are important in the sciences and arise from the concept of power series. In addition, they allow the formulation of an analysis involving differentiation and integration, a requirement for the expression of even simple laws of nature.

While the first two properties are readily satisfied by the rational numbers, the geometric requirements demand using at least the set of algebraic numbers. Transcendental functions, being the result of limiting processes, require Cauchy

2000 Mathematics Subject Classification. 26E30, 12J25, 32P05, 11D88, 46 S10.

Key words and phrases. non-Archimedean analysis, Levi-Civita fields, power series, measure theory and integration, optimization, existence and uniqueness of solutions of ODEs, computational applications.

(C)2010 American Mathematical Society 
completeness, and it is easily shown that the field of real numbers $\mathbb{R}$ is the smallest ordered field having this property.

$\mathbb{R}$ is Archimedean; that is if $x, y \in \mathbb{R}$ are such that $0<|x|<|y|$ then we can find a positive integer $n$ such that $|n x|>|y|$. This Archimedean property of $\mathbb{R}$ corresponds to common sense experiences of measurement: we can measure distances as small as we want; and if one distance is smaller than another, then by taking enough copies of the smaller distance and stacking them end to end, we can eventually produce something which exceeds the bigger distance. Since any Archimedean Cauchy-complete field is isomorphic to $\mathbb{R}$, it is indeed this property that makes the real numbers unique. However, the Archimedean axiom breaks down at the Planck scale, that is for distances less than $1.6 \times 10^{-35}$ meters and durations less than $5.4 \times 10^{-44}$ seconds. Despite our entrenched belief that space and time are continuous, homogeneous, infinitely divisible quantities, we are confronted with the fact that below the Planck scale, distances and durations cannot be scaled up to produce macroscopic ones. Equivalently, we cannot meaningfully measure distances or durations below that scale.

Moreover, while the field $\mathbb{R}$ and its algebraic completion $\mathbb{C}$ as well as the vector space $\mathbb{R}^{n}$ have certainly proven extremely successful for the expression and rigorous mathematical formulation of many physical concepts, they have two shortcomings in interpreting intuitive scientific concepts. First, they do not permit a direct representation of improper functions such as those used for the description of point charges; of course, within the framework of distributions, these concepts can be accounted for in a rigorous fashion, but at the expense of the intuitive interpretation. Second, another intuitive concept of the fathers of analysis, and for that matter quite a number of modern scientists sacrificing rigor for intuition, the idea of derivatives as differential quotients, that is slopes of secants with infinitely small abscissa and ordinate differences cannot be formulated rigorously within $\mathbb{R}$. Especially for the purpose of computational differentiation, the concept of "derivatives are differential quotients" would of course be a remedy to many problems, since it would replace any attempted limiting process involving the unavoidable cancelation of digits by computer-friendly algebra in a new number system.

Since the "fine structure" of the continuum is not observable by means of science, Archimedicity is not required by nature, and leaving it behind may possibly provide solutions for the problems mentioned in the preceding paragraphs and allow a better understanding of the universe. So it appears intriguing to study nonArchimedean field extensions of $\mathbb{R}$, as long as the essential properties of the real numbers mentioned above are preserved.

There are simple ways to construct non-Archimedean extensions of the real numbers (see for example the books of Rudin [36], Hewitt and Stromberg [18], or Stromberg [50], or at a deeper level the works of Fuchs [15], Ebbinghaus et al. [13] or Lightstone and Robinson [28]), but such extensions usually quickly fail to satisfy one or several of the above criteria of a "useful" field, often already regarding the universal existence of roots.

An important idea for the problem of the infinite came from Schmieden and Laugwitz [38], which was then quickly applied to delta functions $[\mathbf{2 1}, \mathbf{2 3}]$ and distributions $[\mathbf{2 2}]$. Certain equivalence classes of sequences of real numbers become the new number set, and, perhaps most interesting, logical statements are considered proved if they hold for "most" of the elements of the sequences. This approach 
lends itself to the introduction of a general scheme that allows the transfer of many properties of the real numbers to the new structure. This method supplies an elegant tool that, in particular, permits the determination of derivatives as differential quotients.

Unfortunately, the resulting structure has two shortcomings. On the one hand, while very large, it is not a field; there are zero divisors, and the ring is not totally ordered. On the other hand, the structure is already so large that individual numbers can never be represented by only a finite amount of information and are thus out of reach for computational problems. Robinson [34] recognized that the intuitive method can be generalized $[\mathbf{2 4}]$ by a nonconstructive process based on model theory to obtain an ordered field, and initiated the branch of Nonstandard Analysis. Some of the standard works describing this field are from Robinson [35], Stroyan and Luxemburg [51], and Davis [12]. In this discipline, the transfer of theorems about real numbers is extremely simple, although at the expense of a nonconstructive process invoking the axiom of choice, leading to an exceedingly large structure of numbers and theorems. The nonconstructiveness makes practical use difficult and leads to several oddities; for example, the fact that the sign of certain elements, although assured to be either positive or negative, can not be decided.

Another approach to a theory of infinitely small numbers originated in game theory and was pioneered by John Conway in his marvel "On Numbers and Games" [11]. A humorous and totally nonstandard yet at the same time very insightful account of these numbers can also be found in Donald Knuth's mathematical novelette "Surreal Numbers: How Two Ex-Students Turned to Pure Mathematics and Found Total Happiness" [19]. Other important accounts on surreal numbers are by Alling $[\mathbf{1}]$ and Gonshor $[\mathbf{1 6}]$.

In the rest of this section, we follow a constructive approach to get the LeviCivita field $\mathcal{R}[\mathbf{2 6}, \mathbf{2 7}]$ as the smallest non-Archimedean ordered field extension of $\mathbb{R}$ that is both real closed and complete in the order topology. Then in the following sections we will review recent research done on $\mathcal{R}$ and its complex counterpart $\mathcal{C}:=\mathcal{R}(i)=\mathcal{R} \oplus i \mathcal{R}$. We note in passing here (and that will become clearer in the next section) that $\mathcal{R}$ and $\mathcal{C}$ are (isomorphic to) subfields of the generalized power series fields $\left[\left[\mathbb{R}^{\mathbb{Q}}\right]\right]$ and $\left[\left[\mathbb{C}^{\mathbb{Q}}\right]\right]$, respectively. We recall that if $F$ is a field and $G$ is an ordered abelian group then the field of generalized power series with exponents in $G$ and coefficients in $F$ is given by

$$
\left[\left[F^{G}\right]\right]:=\left\{\sum_{g \in G} a_{g} X^{g}:\left\{g: a_{g} \neq 0\right\} \text { is a well-ordered subset of } G\right\} ;
$$

it is equipped with the natural addition and multiplication, and if $F$ is an ordered field then $\left[\left[F^{G}\right]\right]$ can be given a lexicographic (total) order. We also recall that the theorem of Hahn [17] entails that any ordered non-Archimedean field extension of $\mathbb{R}$ is isomorphic to a subfield of a generalized power series field $\left[\left[\mathbb{R}^{G}\right]\right]$ for some ordered abelian group $G$.

Any ordered non-Archimedean ring or field extension $E$ of $\mathbb{R}$ has to be infinite dimensional when viewed as a vector space over $\mathbb{R}$. For if $d$ is an infinitely small number in $E$ then the set $\left\{d^{n}: n \in \mathbb{N}\right\}$ is linearly independent over $\mathbb{R}$. Thus the ring $\mathbb{R}[[X]]$ of formal power series over $\mathbb{R}$, equipped with a lexicographic order, is 
the smallest such extension; but $\mathbb{R}[[X]]$ is not a field: a formal power series has a multiplicative inverse if and only if the constant term is not zero.

The smallest field that contains $\mathbb{R}[[X]]$ is the quotient field or the field of fractions of formal power series over $\mathbb{R}$ which is isomorphic to the field of formal Laurent series over $\mathbb{R}$, denoted by $\mathbb{R}((X))$; it is also isomorphic to the field of generalized power series $\left[\left[\mathbb{R}^{\mathbb{Z}}\right]\right]$.

The formal Laurent series field $\left[\left[\mathbb{R}^{\mathbb{Z}}\right]\right]$ is neither real closed nor Cauchy complete in the topology induced by the order. Its real closure is the field of Puiseux series with ascending powers over $\mathbb{R}$; and it is equal to $\bigcup_{n \in \mathbb{N}}\left[\left[\mathbb{R}^{\left(\frac{1}{n} \mathbb{Z}\right)}\right]\right]$. However, the Puiseux series field is not Cauchy complete. Next we will show that $\mathcal{R}$ is the smallest ordered field extension of $\mathbb{R}$ as well as of the formal power series ring, the formal Laurent series field and the Puiseux series field, which is both real closed and complete in the order topology.

First we note that the generalized power series field $\left[\left[\mathbb{R}^{\mathbb{Q}}\right]\right]$ is real closed by $[\mathbf{3 3}]$ (6.10); and it follows from $[\mathbf{2}]$ (Theorem 2.11 on Page 34 ) that $\left[\left[\mathbb{C}^{\mathbb{Q}}\right]\right]$ is algebraically closed. Now consider a polynomial $P$ of degree $n$ over $\mathcal{C}$; then the coefficients of $P$ all have left-finite supports. Since $\left[\left[\mathbb{C}^{\mathbb{Q}}\right]\right]$ is algebraically closed, $P$ (viewed as a polynomial over $\left[\left[\mathbb{C}^{\mathbb{Q}}\right]\right]$ ) has $n$ roots in $\left[\left[\mathbb{C}^{\mathbb{Q}}\right]\right]$ (counting the multiplicities of the roots). Using induction on the degree of the polynomial $P$ and the fact that the coefficients of $P$ have left-finite supports, one can easily show that all the $n$ roots of $P$ must be in $\mathcal{C}$. Thus, $\mathcal{C}$ is algebraically closed; and hence $\mathcal{R}$ is real closed, again by [2] (Theorem 2.11).

Moreover, it is shown in [4] that $\mathcal{R}$ is Cauchy complete in the order topology and that it is the smallest such non-Archimedean field extension of $\mathbb{R}$ that is also real closed. Using that and the inclusions

$$
\mathbb{R} \subset \mathbb{R}[[X]] \subset\left[\left[\mathbb{R}^{\mathbb{Z}}\right]\right] \subset \bigcup_{n \in \mathbb{N}}\left[\left[\mathbb{R}^{\left(\frac{1}{n} \mathbb{Z}\right)}\right]\right] \subset \mathcal{R},
$$

we finish the proof of the statement above.

\section{The fields $\mathcal{R}$ and $\mathcal{C}$}

In this section, we review the algebraic structure of $\mathcal{R}$ and $\mathcal{C}$. We recall that the elements of $\mathcal{R}$ and $\mathcal{C}$ are functions from $\mathbb{Q}$ to $\mathbb{R}$ and $\mathbb{C}$, respectively, with leftfinite support (denoted by supp). That is, below every rational number $q$, there are only finitely many points where the given function does not vanish. For the further discussion, it is convenient to introduce the following terminology.

Definition 2.1. $\left(\lambda, \sim, \approx,=_{r}\right)$ For $x \neq 0$ in $\mathcal{R}$ or $\mathcal{C}$, we let $\lambda(x)=\min (\operatorname{supp}(x))$, which exists because of the left-finiteness of $\operatorname{supp}(x)$; and we let $\lambda(0)=+\infty$.

Given $x, y \in \mathcal{R}$ or $\mathcal{C}$ and $r \in \mathbb{R}$, we say $x \sim y$ if $\lambda(x)=\lambda(y) ; x \approx y$ if $\lambda(x)=\lambda(y)$ and $x[\lambda(x)]=y[\lambda(y)]$; and $x={ }_{r} y$ if $x[q]=y[q]$ for all $q \leq r$.

At this point, these definitions may feel somewhat arbitrary; but after having introduced an order on $\mathcal{R}$, we will see that $\lambda$ describes orders of magnitude, the relation $\approx$ corresponds to agreement up to infinitely small relative error, while $\sim$ corresponds to agreement of order of magnitude.

The sets $\mathcal{R}$ and $\mathcal{C}$ are endowed with formal power series multiplication and componentwise addition, which make them into fields $[4]$ in which we can isomorphically embed $\mathbb{R}$ and $\mathbb{C}$ (respectively) as subfields via the map $\Pi: \mathbb{R}, \mathbb{C} \rightarrow \mathcal{R}, \mathcal{C}$ 
defined by

$$
\Pi(x)[q]=\left\{\begin{array}{ll}
x & \text { if } q=0 \\
0 & \text { else }
\end{array} .\right.
$$

Definition 2.2. (Order in $\mathcal{R}$ ) Let $x \neq y$ in $\mathcal{R}$ be given. Then we say $x>y$ if $(x-y)[\lambda(x-y)]>0$; furthermore, we say $x<y$ if $y>x$.

With this definition of the order relation, $\mathcal{R}$ is an ordered field. Moreover, the embedding $\Pi$ in Equation (2.1) of $\mathbb{R}$ into $\mathcal{R}$ is compatible with the order. The order induces an absolute value on $\mathcal{R}$, from which an absolute value on $\mathcal{C}$ is obtained in the natural way: $|x+i y|=\sqrt{x^{2}+y^{2}}$. We also note here that $\lambda$, as defined above, is a valuation; moreover, the relation $\sim$ is an equivalence relation, and the set of equivalence classes (the value group) is (isomorphic to) $\mathbb{Q}$.

Besides the usual order relations, some other notations are convenient.

Definition 2.3. $(\ll, \gg)$ Let $x, y \in \mathcal{R}$ be non-negative. We say $x$ is infinitely smaller than $y$ (and write $x \ll y$ ) if $n x<y$ for all $n \in \mathbb{N}$; we say $x$ is infinitely larger than $y$ (and write $x \gg y$ ) if $y \ll x$. If $x \ll 1$, we say $x$ is infinitely small; if $x \gg 1$, we say $x$ is infinitely large. Infinitely small numbers are also called infinitesimals or differentials. Infinitely large numbers are also called infinite. Non-negative numbers that are neither infinitely small nor infinitely large are also called finite.

Definition 2.4. (The Number $d$ ) Let $d$ be the element of $\mathcal{R}$ given by $d[1]=1$ and $d[q]=0$ for $q \neq 1$.

It is easy to check that $d^{q} \ll 1$ if $q>0$ and $d^{q} \gg 1$ if $q<0$. Moreover, for all $x \in \mathcal{R}$ (resp. $\mathcal{C}$ ), the elements of $\operatorname{supp}(x)$ can be arranged in ascending order, say $\operatorname{supp}(x)=\left\{q_{1}, q_{2}, \ldots\right\}$ with $q_{j}<q_{j+1}$ for all $j$; and $x$ can be written as $x=\sum_{j=1}^{\infty} x\left[q_{j}\right] d^{q_{j}}$, where the series converges in the topology induced by the absolute value [4].

Altogether, it follows that $\mathcal{R}$ and $\mathcal{C}$ are non-Archimedean field extensions of $\mathbb{R}$ and $\mathbb{C}$, respectively. For a detailed study of these fields, we refer the reader to $[4,40,39,6,5,41,42,48,43,7,44,49,45,46,47]$. In particular, it is shown that $\mathcal{R}$ and $\mathcal{C}$ are complete with respect to the topology induced by the absolute value. In the wider context of valuation theory, it is interesting to note that the topology induced by the absolute value is the same as that introduced via the valuation $\lambda$, as was shown in [45].

It follows therefore that the fields $\mathcal{R}$ and $\mathcal{C}$ are just special cases of the class of fields discussed in [37]. For a general overview of the algebraic properties of generalized power series fields in general, we refer the reader to the comprehensive overview by Ribenboim [33], and for an overview of the related valuation theory to the books by Krull [20], Schikhof [37] and Alling [1]. A thorough and complete treatment of ordered structures can also be found in [32].

Besides being the smallest ordered non-Archimedean field extension of the real numbers that is both complete in the order topology and real closed, the LeviCivita field $\mathcal{R}$ is of particular interest because of its practical usefulness. Since the supports of the elements of $\mathcal{R}$ are left-finite, it is possible to represent these numbers on a computer [4]. Having infinitely small numbers, the errors in classical numerical methods can be made infinitely small and hence irrelevant in all practical 
applications. One such application is the computation of derivatives of real functions representable on a computer $[\mathbf{4 0 , 4 2}]$, where both the accuracy of formula manipulators and the speed of classical numerical methods are achieved.

In the following sections, we present a brief overview of recent research done on $\mathcal{R}$ and $\mathcal{C}$; and we refer the interested reader to the respective papers for a more detailed study of any of the research topics summarized below.

\section{Calculus on $\mathcal{R}$}

The following examples show that functions on a finite interval of $\mathcal{R}$ behave in a way that is different from (and even opposite to) what we would expect under similar conditions in $\mathbb{R}$.

ExAMPLE 3.1. Let $f_{1}:[0,1] \rightarrow \mathcal{R}$ be given by

$$
f_{1}(x)= \begin{cases}d^{-1} & \text { if } 0 \leq x<d \\ d^{-1 / \lambda(x)} & \text { if } d \leq x \ll 1 \\ 1 & \text { if } x \sim 1\end{cases}
$$

Then $f_{1}$ is continuous on $[0,1]$; but for $d \leq x \ll 1, f_{1}(x)$ grows without bound.

EXAMPLE 3.2. Let $f_{2}:[-1,1] \rightarrow \mathcal{R}$ be given by

$$
f_{2}(x)=x-x[0] .
$$

Then $f_{2}$ is continuous on $[-1,1]$. However, $f_{2}$ assumes neither a maximum nor a minimum on $[-1,1]$. The set $f_{2}([-1,1])$ is bounded above by any positive real number and below by any negative real number; but it has neither a least upper bound nor a greatest lower bound.

ExAMPLE 3.3. Let $f_{3}:[0,1] \rightarrow \mathcal{R}$ be given by

$$
f_{3}(x)=\left\{\begin{array}{ll}
1 & \text { if } x \sim 1 \\
0 & \text { if } x \ll 1
\end{array} .\right.
$$

Then $f_{3}$ is continuous on $[0,1]$ and differentiable on $(0,1)$, with $f_{3}^{\prime}(x)=0$ for all $x \in(0,1)$. We have that $f_{3}(0)=0$ and $f_{3}(1)=1$; but $f_{3}(x) \neq 1 / 2$ for all $x \in[0,1]$. Moreover, $f_{3}$ is not constant on $[0,1]$ even though $f_{3}^{\prime}(x)=0$ for all $x \in(0,1)$.

ExAMPLE 3.4. Let $f_{4}:[-1,1] \rightarrow \mathcal{R}$ be given by

$$
f_{4}(x)=\sum_{\nu=1}^{\infty} x_{\nu} d^{3 q_{\nu}} \text { when } x=x[0]+\sum_{\nu=1}^{\infty} x_{\nu} d^{q_{\nu}} .
$$

Then $f_{4}^{\prime}(x)=0$ for all $x \in(-1,1)$. But $f_{4}$ is not constant on $[-1,1]$.

EXAMPLE 3.5. Let $f_{5}:(-1,1) \rightarrow \mathcal{R}$ be given by

$$
f_{5}(x)=-f_{4}(x)+x^{4}
$$

where $f_{4}$ is the function from Example 3.4. Then $f_{5}^{\prime}(x)=4 x^{3}$ for all $x \in(-1,1)$. Thus, $f_{5}^{\prime}>0$ on $(0,1)$; but $f_{5}$ is strictly decreasing on the subset $\{x: 0<x \ll 1\}$ of $(-1,1)$. Also $f_{5}^{\prime}$ is strictly increasing and $f_{5}^{\prime \prime} \geq 0$ on $(-1,1)$; but $f_{5}$ is not convex on $(-1,1)$ since $f_{5}(d)=-d^{3}+d^{4}<0=f_{5}(0)+f_{5}^{\prime}(0) d$. 
ExAmple 3.6. Let $f_{6}:(-1,1) \rightarrow \mathcal{R}$ be given by

$$
f_{6}(x)=-\left(f_{4}(x)\right)^{2}+x^{8},
$$

where $f_{4}$ is again the function from Example 3.4. Then $f_{6}$ is infinitely often differentiable on $(-1,1)$ with $f_{6}^{(j)}(0)=0$ for $1 \leq j \leq 7$ and $f_{6}^{(8)}(0)=8$ ! $>0$. But $f_{6}$ has a relative maximum at 0 .

The difficulties embodied in the examples above are not specific to $\mathcal{R}$, but are common to all non-Archimedean ordered fields; and they result from the fact that $\mathcal{R}$ is disconnected in the topology induced by the order. This makes developing Analysis on the field more difficult than in the real case; for example, the existence of nonconstant functions whose derivatives vanish everywhere on an interval (as in example 3.4) makes integration much harder and renders the solutions of the simplest initial value problems (e.g. $y^{\prime}=0 ; y(0)=0$ ) not unique. To circumvent such difficulties, different approaches have been employed. For example, by imposing stronger conditions on the function than in the real case, the function satisfies an intermediate value theorem and an inverse function theorem [43]; by using a stronger concept of continuity and differentiability than in the real case, one-dimensional and multi-dimensional optimization results similar to those from Real Analysis have been obtained for $\mathcal{R}$-valued functions $[\mathbf{4 8}, 49]$; by carefully defining a measure on $\mathcal{R}$ in [44], we succeed in developing an integration theory with similar properties to those of the Lebesgue integral of Real Analysis; and by restricting solutions of initial value problems to analytic functions, the uniqueness of the solutions will be assured [10].

\section{Review of Power Series and $\mathcal{R}$-Analytic Functions}

Power series on the Levi-Civita field $\mathcal{R}$ have been studied in details in [39, 41, 45, 46, 47]; work prior to that has been mostly restricted to power series with real coefficients. In $[\mathbf{2 6}, \mathbf{2 7}, \mathbf{3 1}, \mathbf{2 5}]$, they could be studied for infinitely small arguments only, while in [4], using the newly introduced weak topology (see Definition 4.4 below), also finite arguments were possible. Moreover, power series over complete valued fields in general have been studied by Schikhof [37], Alling [1] and others in valuation theory, but always in the valuation topology.

In [41], we study the general case when the coefficients in the power series are Levi-Civita numbers (i.e. elements of $\mathcal{R}$ or $\mathcal{C}$ ), using the weak convergence of [4]. We derive convergence criteria for power series which allow us to define a radius of convergence $\eta$ such that the power series converges weakly for all points whose distance from the center is smaller than $\eta$ by a finite amount and it converges in the order topology for all points whose distance from the center is infinitely smaller than $\eta$.

In [45] it is shown that, within their radius of convergence, power series are infinitely often differentiable and the derivatives to any order are obtained by differentiating the power series term by term. Also, power series can be re-expanded around any point in their domain of convergence and the radius of convergence of the new series is equal to the difference between the radius of convergence of the original series and the distance between the original and new centers of the series.

In the following, we summarize some of the key results in $[\mathbf{4 1}, \mathbf{4 5}, \mathbf{4 6}, \mathbf{4 7}]$. We start with a brief review of the convergence of sequences in two different topologies. 
Definition 4.1. A sequence $\left(s_{n}\right)$ in $\mathcal{R}$ or $\mathcal{C}$ is called regular if the union of the supports of all members of the sequence is a left-finite subset of $\mathbb{Q}$. (Recall that $A \subset \mathbb{Q}$ is said to be left-finite if for every $q \in \mathbb{Q}$ there are only finitely many elements in $A$ that are smaller than q.)

Definition 4.2. We say that a sequence $\left(s_{n}\right)$ converges strongly in $\mathcal{R}$ or $\mathcal{C}$ if it converges with respect to the topology induced by the absolute value.

As we have already mentioned in Section 2, strong convergence is equivalent to convergence in the topology induced by the valuation $\lambda$. It is shown in $[\mathbf{3}]$ that the fields $\mathcal{R}$ and $\mathcal{C}$ are complete with respect to the strong topology; and a detailed study of strong convergence can be found in $[39,41]$.

Since power series with real (complex) coefficients do not converge strongly for any nonzero real (complex) argument, it is advantageous to study a new kind of convergence. We do that by defining a family of semi-norms on $\mathcal{R}$ or $\mathcal{C}$, which induces a topology weaker than the topology induced by the absolute value and called weak topology.

Definition 4.3. Given $r \in \mathbb{R}$, we define a mapping $\|\cdot\|_{r}: \mathcal{R}$ or $\mathcal{C} \rightarrow \mathbb{R}$ as follows: $\|x\|_{r}=\max \{|x[q]|: q \in \mathbb{Q}$ and $q \leq r\}$.

The maximum in Definition 4.3 exists in $\mathbb{R}$ since, for any $r \in \mathbb{R}$, only finitely many of the $x[q]$ 's considered do not vanish.

Definition 4.4. A sequence $\left(s_{n}\right)$ in $\mathcal{R}$ (resp. $\mathcal{C}$ ) is said to be weakly convergent if there exists $s \in \mathcal{R}$ (resp. $\mathcal{C}$ ), called the weak limit of the sequence $\left(s_{n}\right)$, such that for all $\epsilon>0$ in $\mathbb{R}$, there exists $N \in \mathbb{N}$ such that $\left\|s_{m}-s\right\|_{1 / \epsilon}<\epsilon$ for all $m \geq N$.

It is shown [4] that $\mathcal{R}$ and $\mathcal{C}$ are not Cauchy complete with respect to the weak topology and that strong convergence implies weak convergence to the same limit. A detailed study of weak convergence is found in $[4,39,41]$.

4.1. Power Series. In the following, we review strong and weak convergence criteria for power series, Theorem 4.5 and Theorem 4.6, the proofs of which are given in [41]. We also note that, since strong convergence is equivalent to convergence with respect to the valuation topology, Theorem 4.5 is a special case of the result on page 59 of $[\mathbf{3 7}]$.

Theorem 4.5. (Strong Convergence Criterion for Power Series) Let $\left(a_{n}\right)$ be a sequence in $\mathcal{R}$ (resp. $\mathcal{C}$ ), and let

$$
\lambda_{0}=\limsup _{n \rightarrow \infty}\left(\frac{-\lambda\left(a_{n}\right)}{n}\right) \text { in } \mathbb{R} \cup\{-\infty, \infty\} \text {. }
$$

Let $x_{0} \in \mathcal{R}$ (resp. $\mathcal{C}$ ) be fixed and let $x \in \mathcal{R}$ (resp. $\mathcal{C}$ ) be given. Then the power series $\sum_{n=0}^{\infty} a_{n}\left(x-x_{0}\right)^{n}$ converges strongly if $\lambda\left(x-x_{0}\right)>\lambda_{0}$ and is strongly divergent if $\lambda\left(x-x_{0}\right)<\lambda_{0}$ or if $\lambda\left(x-x_{0}\right)=\lambda_{0}$ and $-\lambda\left(a_{n}\right) / n>\lambda_{0}$ for infinitely many $n$.

Theorem 4.6. (Weak Convergence Criterion for Power Series) Let $\left(a_{n}\right)$ be a sequence in $\mathcal{R}$ (resp. $\mathcal{C}$ ), and let $\lambda_{0}=\limsup _{n \rightarrow \infty}\left(-\lambda\left(a_{n}\right) / n\right) \in \mathbb{Q}$. Let $x_{0} \in \mathcal{R}$ (resp. $\mathcal{C}$ ) be fixed, and let $x \in \mathcal{R}$ (resp. $\mathcal{C})$ be such that $\lambda\left(x-x_{0}\right)=\lambda_{0}$. For each $n \geq 0$, let $b_{n}=a_{n} d^{n \lambda_{0}}$. Suppose that the sequence $\left(b_{n}\right)$ is regular and write 
$\bigcup_{n=0}^{\infty} \operatorname{supp}\left(b_{n}\right)=\left\{q_{1}, q_{2}, \ldots\right\}$; with $q_{j_{1}}<q_{j_{2}}$ if $j_{1}<j_{2}$. For each $n$, write $b_{n}=$ $\sum_{j=1}^{\infty} b_{n_{j}} d^{q_{j}}$, where $b_{n_{j}}=b_{n}\left[q_{j}\right]$. Let

$$
\eta=\frac{1}{\sup \left\{\lim \sup _{n \rightarrow \infty}\left|b_{n_{j}}\right|^{1 / n}: j \geq 1\right\}} \text { in } \mathbb{R} \cup\{\infty\},
$$

with the conventions $1 / 0=\infty$ and $1 / \infty=0$. Then $\sum_{n=0}^{\infty} a_{n}\left(x-x_{0}\right)^{n}$ converges absolutely weakly if $\left|\left(x-x_{0}\right)\left[\lambda_{0}\right]\right|<\eta$ and is weakly divergent if $\left|\left(x-x_{0}\right)\left[\lambda_{0}\right]\right|>\eta$.

REMARK 4.7. The number $\eta$ in Equation (4.1) is referred to as the radius of weak convergence of the power series $\sum_{n=0}^{\infty} a_{n}\left(x-x_{0}\right)^{n}$.

As an immediate consequence of Theorem 4.6, we obtain the following result which allows us to extend real and complex functions representable by power series to the Levi-Civita fields $\mathcal{R}$ and $\mathcal{C}$. This result is of particular interest for the application discussed in Section 7.

Corollary 4.8. (Power Series with Purely Real or Complex Coefficients) Let $\sum_{n=0}^{\infty} a_{n} X^{n}$ be a power series with purely real (resp. complex) coefficients and with classical radius of convergence equal to $\eta$. Let $x \in \mathcal{R}$ (resp. $\mathcal{C}$ ), and let $A_{n}(x)=\sum_{j=0}^{n} a_{j} x^{j} \in \mathcal{R}$ (resp. $\left.\mathcal{C}\right)$. Then, for $|x|<\eta$ and $|x| \not \approx \eta$, the sequence $\left(A_{n}(x)\right)$ converges absolutely weakly. We define the limit to be the continuation of the power series to $\mathcal{R}$ (resp. $\mathcal{C}$ ).

4.2. $\mathcal{R}$-Analytic Functions. In this section, we review the algebraic and analytical properties of a class of functions that are given locally by power series and we refer the reader to $[\mathbf{4 5}]$ for a more detailed study.

Definition 4.9. Let $a, b \in \mathcal{R}$ be such that $0<b-a \sim 1$ and let $f:[a, b] \rightarrow \mathcal{R}$. Then we say that $f$ is expandable or $\mathcal{R}$-analytic on $[a, b]$ if for all $x \in[a, b]$ there exists a finite $\delta>0$ in $\mathcal{R}$, and there exists a regular sequence $\left(a_{n}(x)\right)$ in $\mathcal{R}$ such that, under weak convergence, $f(y)=\sum_{n=0}^{\infty} a_{n}(x)(y-x)^{n}$ for all $y \in(x-\delta, x+\delta) \cap$ $[a, b]$.

Definition 4.10. Let $a<b$ in $\mathcal{R}$ be such that $t=\lambda(b-a) \neq 0$ and let $f:[a, b] \rightarrow \mathcal{R}$. Then we say that $f$ is $\mathcal{R}$-analytic on $[a, b]$ if the function $F$ : $\left[d^{-t} a, d^{-t} b\right] \rightarrow \mathcal{R}$, given by $F(x)=f\left(d^{t} x\right)$, is $\mathcal{R}$-analytic on $\left[d^{-t} a, d^{-t} b\right]$.

It is shown in [45] that if $f$ is $\mathcal{R}$-analytic on $[a, b]$ then $f$ is bounded on $[a, b]$; also, if $g$ is $\mathcal{R}$-analytic on $[a, b]$ and $\alpha \in \mathcal{R}$ then $f+\alpha g$ and $f \cdot g$ are $\mathcal{R}$-analytic on $[a, b]$. Moreover, the composition of $\mathcal{R}$-analytic functions is $\mathcal{R}$-analytic. Finally, using the fact that power series on $\mathcal{R}$ are infinitely often differentiable within their domain of convergence and the derivatives to any order are obtained by differentiating the power series term by term $[\mathbf{4 5}]$, we obtain the following result.

TheOREM 4.11. Let $a<b$ in $\mathcal{R}$ be given, and let $f:[a, b] \rightarrow \mathcal{R}$ be $\mathcal{R}$-analytic on $[a, b]$. Then $f$ is infinitely often differentiable on $[a, b]$, and for any positive integer $m$, we have that $f^{(m)}$ is $\mathcal{R}$-analytic on $[a, b]$. Moreover, if $f$ is given locally around $x_{0} \in[a, b]$ by $f(x)=\sum_{n=0}^{\infty} a_{n}\left(x_{0}\right)\left(x-x_{0}\right)^{n}$, then $f^{(m)}$ is given by

$$
f^{(m)}(x)=\sum_{n=m}^{\infty} n(n-1) \cdots(n-m+1) a_{n}\left(x_{0}\right)\left(x-x_{0}\right)^{n-m} .
$$

In particular, we have that $a_{m}\left(x_{0}\right)=f^{(m)}\left(x_{0}\right) / m$ ! for all $m=0,1,2, \ldots$ 
In $[47]$, we focus on the proof of the intermediate value theorem for $\mathcal{R}$-analytic functions (functions that are given locally by power series). Given a function $f$ that is $\mathcal{R}$-analytic on an interval $[a, b]$ and a value $S$ between $f(a)$ and $f(b)$, we use iteration to construct a sequence of numbers in $[a, b]$ that converges in the order topology to a point $c \in[a, b]$ such that $f(c)=S$. The proof is quite involved, making use of many of the results proved in $[4 \mathbf{4 1} 4 \mathbf{4 5}$ as well as some results from Real Analysis, including the intermediate value theorem for real-valued functions, continuous on closed and finite real intervals.

Ongoing research aims at proving the Extreme Value Theorem for $\mathcal{R}$-analytic functions, stated as a conjecture below. Once this conjecture has been proved, the Mean Value Theorem will follow readily.

Conjecture 4.12. (Extreme Value Theorem) Let $a<b$ in $\mathcal{R}$ be given, and let $f:[a, b] \rightarrow \mathcal{R}$ be $\mathcal{R}$-analytic on $[a, b]$. Then $f$ assumes a maximum and a minimum on $[a, b]$.

Finally, in $[\mathbf{4 6}]$ we generalize the results in $[\mathbf{4 1}, \mathbf{4 5}, 4 \mathbf{4 7}]$ to power series with rational exponents over $\mathcal{R}$.

\section{Measure Theory and Integration}

Before we define a measure on $\mathcal{R}$, we introduce the following notations which will be adopted throughout this section: $I(a, b)$ will be used to denote any one of the intervals $[a, b],(a, b],[a, b)$ or $(a, b)$, unless we explicitly specify a particular choice of one of the four intervals. Also, to denote the length of a given interval $I$, we will use the notation $l(I)$.

Definition 5.1. Let $A \subset \mathcal{R}$ be given. Then we say that $A$ is measurable if for every $\epsilon>0$ in $\mathcal{R}$, there exist a sequence of mutually disjoint intervals $\left(I_{n}\right)$ and a sequence of mutually disjoint intervals $\left(J_{n}\right)$ such that $\cup_{n=1}^{\infty} I_{n} \subset A \subset \cup_{n=1}^{\infty} J_{n}$, $\sum_{n=1}^{\infty} l\left(I_{n}\right)$ and $\sum_{n=1}^{\infty} l\left(J_{n}\right)$ converge in $\mathcal{R}$, and $\sum_{n=1}^{\infty} l\left(J_{n}\right)-\sum_{n=1}^{\infty} l\left(I_{n}\right) \leq \epsilon$.

Given a measurable set $A$, then for every $k \in \mathbb{N}$, we can select a sequence of mutually disjoint intervals $\left(I_{n}^{k}\right)$ and a sequence of mutually disjoint intervals $\left(J_{n}^{k}\right)$ such that $\sum_{n=1}^{\infty} l\left(I_{n}^{k}\right)$ and $\sum_{n=1}^{\infty} l\left(J_{n}^{k}\right)$ converge in $\mathcal{R}$ for all $k$,

$$
\cup_{n=1}^{\infty} I_{n}^{k} \subset \cup_{n=1}^{\infty} I_{n}^{k+1} \subset A \subset \cup_{n=1}^{\infty} J_{n}^{k+1} \subset \cup_{n=1}^{\infty} J_{n}^{k} \text { and } \sum_{n=1}^{\infty} l\left(J_{n}^{k}\right)-\sum_{n=1}^{\infty} l\left(I_{n}^{k}\right) \leq d^{k}
$$

for all $k \in \mathbb{N}$. Since $\mathcal{R}$ is Cauchy-complete in the order topology, it follows that $\lim _{k \rightarrow \infty} \sum_{n=1}^{\infty} l\left(I_{n}^{k}\right)$ and $\lim _{k \rightarrow \infty} \sum_{n=1}^{\infty} l\left(J_{n}^{k}\right)$ both exist and they are equal. We call the common value of the limits the measure of $A$ and we denote it by $m(A)$. Thus,

$$
m(A)=\lim _{k \rightarrow \infty} \sum_{n=1}^{\infty} l\left(I_{n}^{k}\right)=\lim _{k \rightarrow \infty} \sum_{n=1}^{\infty} l\left(J_{n}^{k}\right) .
$$

Moreover, since the sequence $\left(\sum_{n=1}^{\infty} l\left(I_{n}^{k}\right)\right)_{k \in \mathbb{N}}$ is nondecreasing and since the sequence $\left(\sum_{n=1}^{\infty} l\left(J_{n}^{k}\right)\right)_{k \in \mathbb{N}}$ is nonincreasing, we have that

$$
\sum_{n=1}^{\infty} l\left(I_{n}^{k}\right) \leq m(A) \leq \sum_{n=1}^{\infty} l\left(J_{n}^{k}\right) \text { for all } k \in \mathbb{N}
$$

This is a free offprint provided to the author by the publisher. Copyright restrictions may apply. 
Contrary to the real case, $\sup \left\{\sum_{n=1}^{\infty} l\left(I_{n}\right): I_{n}\right.$ 's are mutually disjoint intervals and $\left.\cup_{n=1}^{\infty} I_{n} \subset A\right\}$ and $\inf \left\{\sum_{n=1}^{\infty} l\left(J_{n}\right): A \subset \cup_{n=1}^{\infty} J_{n}\right\}$ need not exist for a given set $A \subset \mathcal{R}$. However, we show in [44] that if $A$ is measurable then both the supremum and infimum exist and they are equal to $m(A)$. This shows that the definition of measurable sets in Definition 5.1 is a natural generalization of the Lebesgue measure of real analysis that corrects for the lack of suprema and infima in non-Archimedean totally ordered fields.

Proposition 5.2. Let $A \subset \mathcal{R}$ be measurable. Then

$$
\begin{aligned}
m(A)=\inf & \left\{\sum_{n=1}^{\infty} l\left(J_{n}\right): \text { for all } n J_{n} \text { is an interval, } A \subset \cup_{n=1}^{\infty} J_{n}\right. \text { and } \\
& \left.\sum_{n=1}^{\infty} l\left(J_{n}\right) \text { converges }\right\} \\
= & \sup \left\{\sum_{n=1}^{\infty} l\left(I_{n}\right): I_{n} \text { 's are mutually disjoint intervals, } \cup_{n=1}^{\infty} I_{n} \subset A\right. \text {, and } \\
& \left.\sum_{n=1}^{\infty} l\left(I_{n}\right) \text { converges }\right\} .
\end{aligned}
$$

We prove that the measure defined above has similar properties to those of the Lebesgue measure of Real Analysis. Namely (see [44] for the details), we show that any subset of a measurable set of measure 0 is itself measurable and has measure 0 . We also show that any countable unions of measurable sets whose measures form a null sequence is measurable and the measure of the union is less than or equal to the sum of the measures of the original sets; moreover, the measure of the union is equal to the sum of the measures of the original sets if the latter are mutually disjoint. Then we show that any finite intersection of measurable sets is also measurable and that the sum of the measures of two measurable sets is equal to the sum of the measures of their union and intersection.

Like in $\mathbb{R}$, we first introduce a family of simple functions on $\mathcal{R}$ from which we obtain a larger family of measurable functions. In the Lebesgue measure theory on $\mathbb{R}$, the simple functions consist only of step functions (piece-wise constant functions); and all measurable functions including all monomials, polynomials and power series are obtained as uniform limits of simple functions. It can be easily shown that in $\mathcal{R}$ the order topology is too strong and none of the monomials can be obtained as a uniform limit of polynomials of lower degrees. So using the step functions as our simple functions would yield a too small class of functions that we can integrate. So we introduce a larger family of simple functions. Here we define such a family of simple functions in an abstract way, which we will use throughout the discussions in this section; and but we give two examples below.

Definition 5.3. Let $a<b$ in $\mathcal{R}$ be given and $\mathcal{S}(a, b)$ a family of functions from $I(a, b)$ to $\mathcal{R}$. Then we say that $\mathcal{S}(a, b)$ is a family of simple functions on $I(a, b)$ if the following are true:

(1) $\mathcal{S}(a, b)$ is an algebra that contains the identity function;

(2) for all $f \in \mathcal{S}(a, b), f$ is Lipschitz on $I(a, b)$ and there exists an antiderivative $F$ of $f$ in $\mathcal{S}(a, b)$; 
(3) for all differentiable $f \in \mathcal{S}(a, b)$, if $f^{\prime}=0$ on $I(a, b)$ then $f$ is constant on $I(a, b)$; moreover, if $f^{\prime} \leq 0$ on $I(a, b)$ then $f$ is nonincreasing on $I(a, b)$.

If $f \in \mathcal{S}(a, b)$, we say that $f$ is simple on $I(a, b)$.

It follows from the first condition in Definition 5.3 that any constant function on $I(a, b)$ is in $\mathcal{S}(a, b)$; moreover, if $f, g \in \mathcal{S}(a, b)$ and if $\alpha \in \mathcal{R}$, then $f+\alpha g \in \mathcal{S}(a, b)$. Also, it follows from the third condition that the anti-derivative in the second condition is unique up to a constant. A close look at Definition 5.3 reveals that the polynomials algebra on $I(a, b)$ is the smallest family of simple functions on $I(a, b)$. Another example is the family of $\mathcal{R}$-analytic functions on $I(a, b)$, which was discussed in Section 4 above.

While the third condition in Definition 5.3 is automatically satisfied in real analysis, this is not the case in $\mathcal{R}$, as shown by Example 3.3 and Example 3.4.

Definition 5.4. Let $A \subset \mathcal{R}$ be a measurable subset of $\mathcal{R}$ and let $f: A \rightarrow \mathcal{R}$ be bounded on $A$. Then we say that $f$ is measurable on $A$ if for all $\epsilon>0$ in $\mathcal{R}$, there exists a sequence of mutually disjoint intervals $\left(I_{n}\right)$ such that $I_{n} \subset A$ for all $n, \sum_{n=1}^{\infty} l\left(I_{n}\right)$ converges in $\mathcal{R}, m(A)-\sum_{n=1}^{\infty} l\left(I_{n}\right) \leq \epsilon$ and $f$ is simple on $I_{n}$ for all $n$.

In $[44]$, we derive a simple characterization of measurable functions and we show that they form an algebra. Then we show that a measurable function is differentiable almost everywhere and that a function measurable on two measurable subsets of $\mathcal{R}$ is also measurable on their union and intersection.

We define the integral of a simple function over an interval $I(a, b)$ and we use that to define the integral of a measurable function $f$ over a measurable set $A$.

Definition 5.5. Let $a<b$ in $\mathcal{R}$, let $f: I(a, b) \rightarrow \mathcal{R}$ be simple on $I(a, b)$, and let $F$ be a simple anti-derivative of $f$ on $I(a, b)$. Then the integral of $f$ over $I(a, b)$ is the $\mathcal{R}$ number

$$
\int_{I(a, b)} f=\lim _{x \rightarrow b} F(x)-\lim _{x \rightarrow a} F(x) .
$$

The limits in Definition 5.5 account for the case when the interval $I(a, b)$ does not include one or both of the end points; and these limits exist since $F$ is Lipschitz on $I(a, b)$.

Now let $A \subset \mathcal{R}$ be measurable, let $f: A \rightarrow \mathcal{R}$ be measurable and let $M$ be a bound for $|f|$ on $A$. Then for every $k \in \mathbb{N}$, there exists a sequence of mutually disjoint intervals $\left(I_{n}^{k}\right)_{n \in \mathbb{N}}$ such that $\cup_{n=1}^{\infty} I_{n}^{k} \subset A, \sum_{n=1}^{\infty} l\left(I_{n}^{k}\right)$ converges, $m(A)-$ $\sum_{n=1}^{\infty} l\left(I_{n}^{k}\right) \leq d^{k}$, and $f$ is simple on $I_{n}^{k}$ for all $n \in \mathbb{N}$. Without loss of generality, we may assume that $I_{n}^{k} \subset I_{n}^{k+1}$ for all $n \in \mathbb{N}$ and for all $k \in \mathbb{N}$. Since $\lim _{n \rightarrow \infty} l\left(I_{n}^{k}\right)=0$, and since $\left|\int_{I_{n}^{k}} f\right| \leq M l\left(I_{n}^{k}\right)$ (proved in [44] for simple functions), it follows that

$$
\lim _{n \rightarrow \infty} \int_{I_{n}^{k}} f=0 \text { for all } k \in \mathbb{N} .
$$

Thus, $\sum_{n=1}^{\infty} \int_{I_{n}^{k}} f$ converges in $\mathcal{R}$ for all $k \in \mathbb{N}[\mathbf{4 1}]$.

Next we show that the sequence $\left(\sum_{n=1}^{\infty} \int_{I_{n}^{k}} f\right)_{k \in \mathbb{N}}$ converges in $\mathcal{R}$. So let $\epsilon>0$ be given in $\mathcal{R}$; and let $K \in \mathbb{N}$ be such that $M d^{K} \leq \epsilon$. Let $k>j \geq K$ be given in 
$\mathbb{N}$. Then $\cup_{n=1}^{\infty} I_{n}^{k} \backslash \cup_{n=1}^{\infty} I_{n}^{j}$ can be written as a union of mutually disjoint intervals, say $\left(I_{n}^{j, k}\right)_{n \in \mathbb{N}}$, such that $\sum_{n=1}^{\infty} l\left(I_{n}^{j, k}\right)$ converges, and

$$
\sum_{n=1}^{\infty} l\left(I_{n}^{j, k}\right)=\sum_{n=1}^{\infty} l\left(I_{n}^{k}\right)-\sum_{n=1}^{\infty} l\left(I_{n}^{j}\right) \leq m(A)-\sum_{n=1}^{\infty} l\left(I_{n}^{j}\right) \leq d^{j} \leq d^{K} .
$$

Thus,

$$
\begin{aligned}
\left|\sum_{n=1}^{\infty} \int_{I_{n}^{k}} f-\sum_{n=1}^{\infty} \int_{I_{n}^{j}} f\right| & =\left|\sum_{n=1}^{\infty} \int_{I_{n}^{j, k}} f\right| \leq \sum_{n=1}^{\infty}\left|\int_{I_{n}^{j, k}} f\right| \\
& \leq \sum_{n=1}^{\infty} M l\left(I_{n}^{j, k}\right)=M \sum_{n=1}^{\infty} l\left(I_{n}^{j, k}\right) \\
& \leq M d^{K} \leq \epsilon,
\end{aligned}
$$

where we have used the fact that an infinite series converges if and only if it converges absolutely [41]. Thus, the sequence $\left(\sum_{n=1}^{\infty} \int_{I_{n}^{k}} f\right)_{k \in \mathbb{N}}$ is Cauchy; and hence it converges in $\mathcal{R}$. We define the unique limit as the integral of $f$ over $A$.

Definition 5.6. Let $A \subset \mathcal{R}$ be measurable and let $f: A \rightarrow \mathcal{R}$ be measurable. Then the integral of $f$ over $A$, denoted by $\int_{A} f$, is given by

$$
\int_{A} f=\lim _{\substack{\sum_{\begin{subarray}{c}{n=1 \\
\cup} }}^{\infty} l\left(I_{n}\right) \rightarrow m(A)} \\
{\left(I_{n}\right) \text { are mutually disjoint }} \\
{f \text { is simple on } I_{n} \forall n}\end{subarray}} \sum_{n=1}^{\infty} \int_{I_{n}} f .
$$

It turns out that the integral in Definition 5.6 satisfies similar properties to those of the Lebesgue integral on $\mathbb{R}$; see $[44]$ for the details. In particular, we prove the linearity property of the integral and that if $|f| \leq M$ on $A$ then $\left|\int_{A} f\right| \leq M m(A)$, where $m(A)$ is the measure of $A$. We also show that the sum of the integrals of a measurable function over two measurable sets is equal to the sum of its integrals over the union and the intersection of the two sets. Moreover, we prove the following theorem.

Theorem 5.7. Let $A \subset \mathcal{R}$ be measurable, let $f: A \rightarrow \mathcal{R}$, for each $k \in \mathbb{N}$ let $f_{k}: A \rightarrow \mathcal{R}$ be measurable on $A$, and let the sequence $\left(f_{k}\right)$ converge uniformly to $f$ on $A$. Then $\lim _{k \rightarrow \infty} \int_{A} f_{k}$ exists. Moreover, if $f$ is measurable on $A$, then $\lim _{k \rightarrow \infty} \int_{A} f_{k}=\int_{A} f$.

\section{Optimization}

In [48], we consider unconstrained one-dimensional optimization on $\mathcal{R}$. We study general optimization questions and derive first and second order necessary and sufficient conditions for the existence of local maxima and minima of a function on a convex subset of $\mathcal{R}$. We show that for first order optimization, the results are similar to the corresponding real ones. However, for second and higher order optimization, we show that conventional differentiability is not strong enough to just extend the real-case results (see Example 3.5 and Example 3.6); and a stronger concept of differentiability, the so-called derivate differentiability (see Definition 6.3 below), is used to solve that difficulty. We also characterize convex functions on convex sets of $\mathcal{R}$ in terms of first and second order derivatives. 
In the following, we review the definitions of derivate continuity and differentiability in one dimension, as well as some related results and we refer the interested reader to $[\mathbf{6}, \mathbf{3 9}]$ for a more detailed study.

Definition 6.1. Let $D \subset \mathcal{R}$ be open and let $f: D \rightarrow \mathcal{R}$. Then we say that $f$ is derivate continuous on $D$ if there exists $M \in \mathcal{R}$, called a Lipschitz constant of $f$ on $D$, such that

$$
\left|\frac{f(y)-f(x)}{y-x}\right| \leq M \text { for all } x \neq y \text { in } D
$$

It follows immediately from Definition 6.1 that if $f: D \rightarrow \mathcal{R}$ is derivate continuous on $D$ then $f$ is uniformly continuous (in the conventional sense) on $D$.

REMARK 6.2. It is clear that the concept of derivate continuity in Definition 6.1 coincides with that of uniform Lipschitz continuity when restricted to $\mathbb{R}$. We chose to call it derivate continuity here so that, after having defined derivate differentiability in Definition 6.3 and higher order derivate differentiability in Definition 6.5 , we can think of derivate continuity as derivate differentiability of "order zero", just as is the case for continuity in $\mathbb{R}$.

Definition 6.3. Let $D \subset \mathcal{R}$ be open, let $f: D \rightarrow \mathcal{R}$ be derivate continuous on $D$, and let $I_{D}$ denote the identity function on $D$. Then we say that $f$ is derivate differentiable on $D$ if for all $x \in D$, the function $\frac{f-f(x)}{I_{D}-x}: D \backslash\{x\} \rightarrow \mathcal{R}$ is derivate continuous on $D \backslash\{x\}$. In this case, the unique continuation of $\frac{f-f(x)}{I_{D}-x}$ to $D$ (see [39]) will be called the first derivate function (or simply the derivate function) of $f$ at $x$ and will be denoted by $F_{1, x}$; moreover, the function value $F_{1, x}(x)$ will be called the derivative of $f$ at $x$ and will be denoted by $f^{\prime}(x)$.

It follows immediately from Definition 6.3 that if $f: D \rightarrow \mathcal{R}$ is derivate differentiable then $f$ is differentiable in the conventional sense; moreover, the two derivatives at any given point of $D$ agree. The following result provides a useful tool for checking the derivate differentiability of functions; we refer the interested reader to $[39,48]$ for its proof.

TheOREM 6.4. Let $D \subset \mathcal{R}$ be open and let $f: D \rightarrow \mathcal{R}$ be derivate continuous on $D$. Suppose there exists $M \in \mathcal{R}$ and there exists a function $g: D \rightarrow \mathcal{R}$ such that

$$
\left|\frac{f(y)-f(x)}{y-x}-g(x)\right| \leq M|y-x| \text { for all } y \neq x \text { in } D \text {. }
$$

Then $f$ is derivate differentiable on $D$, with derivative $f^{\prime}=g$.

Definition 6.5 ( $n$-times Derivate Differentiability). Let $D \subset \mathcal{R}$ be open, and let $f: D \rightarrow \mathcal{R}$. Let $n \geq 2$ be given in $\mathbb{N}$. Then we define $n$-times derivate differentiability of $f$ on $D$ inductively as follows: Having defined $(n-1)$-times derivate differentiability, we say that $f$ is $n$-times derivate differentiable on $D$ if $f$ is $(n-1)$-times derivate differentiable on $D$ and for all $x \in D$, the $(n-1)$ st derivate function $F_{n-1, x}$ is derivate differentiable on $D$. For all $x \in D$, the derivate function $F_{n, x}$ of $F_{n-1, x}$ at $x$ will be called the $n$th derivate function of $f$ at $x$, and the number $f^{(n)}(x)=n ! F_{n-1, x}^{\prime}(x)$ will be called the $n$th derivative of $f$ at $x$ and denoted by $f^{(n)}(x)$.

One of the most useful consequences of the derivate differentiability concept is that it gives rise to a Taylor formula with remainder while the conventional 
(topological) differentiability does not. We only state the result here and refer the reader to $[\mathbf{6}, 39]$ for its proof. We also note that, as an immediate result of Theorem 6.6, we obtain local expandability in Taylor series around $x_{0} \in D$ of a given function that is infinitely often derivate differentiable on $D[\mathbf{6}, \mathbf{3 9}]$.

Theorem 6.6 (Taylor Formula with Remainder). Let $D \subset \mathcal{R}$ be open and let $f: D \rightarrow \mathcal{R}$ be $n$-times derivate differentiable on $D$. Let $x \in D$ be given, let $F_{n, x}$ be the nth order derivate function of $f$ at $x$, and let $M_{n, x}$ be a Lipschitz constant of $F_{n, x}$ on $D$. Then for all $y \in D$, we have that

$$
f(y)=f(x)+\sum_{j=1}^{n} \frac{f^{(j)}(x)}{j !}(y-x)^{j}+r_{n+1}(x, y)(y-x)^{n+1},
$$

with $\lambda\left(r_{n+1}(x, y)\right) \geq \lambda\left(M_{n, x}\right)$.

Using Theorem 6.6, we are able to generalize in [48] most of one-dimensional optimization results of Real Analysis. For example, we obtain the following two results which state necessary and sufficient conditions for the existence of local (relative) extrema.

Theorem 6.7 (Necessary Conditions for Existence of Local Extrema). Let $a<b$ be given in $\mathcal{N}$, let $m \geq 2$, and let $f: I(a, b) \rightarrow \mathcal{N}$ be $m$-times derivate differentiable on $I(a, b)$. Suppose that $f$ has a local extremum at $x_{0} \in(a, b)$ and $l \leq m$ is the order of the first nonvanishing derivative of $f$ at $x_{0}$. Then $l$ is even. Moreover, $f^{(l)}\left(x_{0}\right)$ is positive if the extremum is a minimum and negative if the extremum is a maximum.

Theorem 6.8 (Sufficient Conditions for Existence of Local Extrema). Let $a<b$ be given in $\mathcal{N}$, let $k \in \mathbb{N}$, and let $f: I(a, b) \rightarrow \mathcal{N}$ be $2 k$-times derivate differentiable on $I(a, b)$. Let $x_{0} \in(a, b)$ be such $f^{(j)}\left(x_{0}\right)=0$ for all $j \in\{1, \ldots, 2 k-1\}$ and $f^{(2 k)}\left(x_{0}\right) \neq 0$. Then $f$ has a local minimum at $x_{0}$ if $f^{(2 k)}\left(x_{0}\right)>0$ and a local maximum if $f^{(2 k)}\left(x_{0}\right)<0$.

In [49], we generalize the concepts of derivate continuity and differentiability to higher dimensions; and this yields a Taylor Formula with a bounded remainder term for $C^{m}$ functions (in the derivate sense) from an open subset of $\mathcal{R}^{n}$ to $\mathcal{R}$.

Theorem 6.9 (Taylor Formula for Functions of Several Variables). Let $D \subset \mathcal{R}^{n}$ be open, let $\vec{x}_{0} \in D$ be given and let $f: D \rightarrow \mathcal{R}$ be $C^{q}$ on $D$. Then there exist $M, \delta>0$ in $\mathcal{R}$ such that $B_{\delta}\left(\vec{x}_{0}\right) \subset D$ and, for all $\vec{x} \in B_{\delta}\left(\vec{x}_{0}\right)$, we have that

$$
\begin{aligned}
f(\vec{x})= & f\left(\vec{x}_{0}\right)+\sum_{j=1}^{q}\left(\frac{1}{j !} \sum_{l_{1}, \ldots, l_{j}=1}^{n}\left(\partial_{l_{1}} \cdots \partial_{l_{j}} f\left(\vec{x}_{0}\right) \pi_{k=1}^{j}\left(x_{l_{k}}-x_{0, l_{k}}\right)\right)\right) \\
& +R_{q+1}\left(\vec{x}_{0}, \vec{x}\right),
\end{aligned}
$$

where $\left|R_{q+1}\left(\vec{x}_{0}, \vec{x}\right)\right| \leq M\left|\vec{x}-\vec{x}_{0}\right|^{q+1}$.

Then we use that to derive necessary and sufficient conditions of second order for the existence of a minimum of an $\mathcal{R}$-valued function on $\mathcal{R}^{n}$ subject to equality and inequality constraints. More specifically, we solve the problem of minimizing a 
function $f: \mathcal{R}^{n} \rightarrow \mathcal{R}$, subject to the following set of constraints:

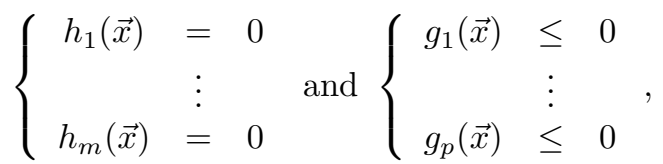

where all the functions in Equation (6.1) are from $\mathcal{R}^{n}$ to $\mathcal{R}$. A point $\overrightarrow{x_{0}} \in \mathcal{R}^{n}$ is said to be a feasible point if it satisfies the constraints in Equation (6.1).

Definition 6.10. Let $\vec{x}_{0}$ be a feasible point for the constraints in Equation (6.1) and let $I\left(\vec{x}_{0}\right)=\left\{l \in\{1, \ldots, p\}: g_{l}\left(\vec{x}_{0}\right)=0\right\}$. Then we say that $\vec{x}_{0}$ is regular for the constraints if $\left\{\nabla h_{j}\left(\vec{x}_{0}\right): j=1, \ldots, m ; \nabla g_{l}\left(\vec{x}_{0}\right): l \in I\left(\vec{x}_{0}\right)\right\}$ forms a linearly independent subset of vectors in $\mathcal{R}^{n}$.

The following theorem provides necessary conditions of second order for a local minimizer $\vec{x}_{0}$ of a function $f$ subject to the constraints in Equation (6.1). The result is a generalization of the corresponding real result $[\mathbf{2 9}, \mathbf{1 4}]$ and the proof (see [49]) is similar to that of the latter; but one essential difference is the form of the remainder formula. In the real case, the remainder term is related to the second derivative at some intermediate point, while here that is not the case. However, the concept of derivate differentiability puts a bound on the remainder term; and this is instrumental in the proof of the theorem.

Theorem 6.11. Suppose that $f,\left\{h_{j}\right\}_{j=1}^{m},\left\{g_{l}\right\}_{l=1}^{p}$ are $C^{2}$ on some open set $D \subset \mathcal{R}^{n}$ containing the point $\vec{x}_{0}$ and that $\vec{x}_{0}$ is a regular point for the constraints in Equation (6.1). If $\vec{x}_{0}$ is a local minimizer for $f$ under the given constraints, then there exist $\alpha_{1}, \ldots, \alpha_{m}, \beta_{1}, \ldots, \beta_{p} \in \mathcal{R}$ such that

(i) $\beta_{l} \geq 0$ for all $l \in\{1, \ldots, p\}$,

(ii) $\beta_{l} g_{l}\left(\vec{x}_{0}\right)=0$ for all $l \in\{1, \ldots, p\}$,

(iii) $\nabla f\left(\vec{x}_{0}\right)+\sum_{j=1}^{m} \alpha_{j} \nabla h_{j}\left(\vec{x}_{0}\right)+\sum_{l=1}^{p} \beta_{l} \nabla g_{l}\left(\vec{x}_{0}\right)=\overrightarrow{0}$, and

(iv) $\vec{y}^{T}\left(\nabla^{2} f\left(\vec{x}_{0}\right)+\sum_{j=1}^{m} \alpha_{j} \nabla^{2} h_{j}\left(\vec{x}_{0}\right)+\sum_{l=1}^{p} \beta_{l} \nabla^{2} g_{l}\left(\vec{x}_{0}\right)\right) \vec{y} \geq 0$ for all $\vec{y} \epsilon$ $\mathcal{R}^{n}$ satisfying $\nabla h_{j}\left(\vec{x}_{0}\right) \vec{y}=0$ for all $j \in\{1, \ldots, m\}, \nabla g_{l}\left(\vec{x}_{0}\right) \vec{y}=0$ for all $l \in L=\left\{k \in I\left(\vec{x}_{0}\right): \beta_{k}>0\right\}$ and $\nabla g_{l}\left(\vec{x}_{0}\right) \vec{y} \leq 0$ for all $l \in I\left(\vec{x}_{0}\right) \backslash L$.

In the following theorem, we present second order sufficient conditions for a feasible point $\vec{x}_{0}$ to be a local minimum of a function $f$ subject to the constraints in Equation (6.1). It is a generalization of the real result [14] and reduces to it, when restricted to functions from $\mathbb{R}^{n}$ to $\mathbb{R}$. In fact, since $\epsilon$ in condition (iv) below is allowed to be infinitely small, the condition $\left|\nabla h_{j}\left(\vec{x}_{0}\right) \vec{y}\right|<\epsilon$ would reduce to $\nabla h_{j}\left(\vec{x}_{0}\right) \vec{y}=0$, when restricted to $\mathbb{R}$. Similarly, one can readily see that the other conditions are mere generalizations of the corresponding real ones. However, the proof (see [49]) is different than that of the real result since the supremum principle does not hold in $\mathcal{R}$.

TheOREM 6.12. Suppose that $f,\left\{h_{j}\right\}_{j=1}^{m},\left\{g_{l}\right\}_{l=1}^{p}$ are $C^{2}$ on some open set $D \subset \mathcal{R}^{n}$ containing the point $\vec{x}_{0}$ and that $\vec{x}_{0}$ is a feasible point for the constraints in Equation (6.1) such that, for some $\alpha_{1}, \ldots, \alpha_{m}, \beta_{1}, \ldots, \beta_{p} \in \mathcal{R}$ and for some $\epsilon, \gamma>0$ in $\mathcal{R}$, we have that

(i) $\beta_{l} \geq 0$ for all $l \in\{1, \ldots, p\}$,

(ii) $\beta_{l} g_{l}\left(\vec{x}_{0}\right)=0$ for all $l \in\{1, \ldots, p\}$,

(iii) $\nabla f\left(\vec{x}_{0}\right)+\sum_{j=1}^{m} \alpha_{j} \nabla h_{j}\left(\vec{x}_{0}\right)+\sum_{l=1}^{p} \beta_{l} \nabla g_{l}\left(\vec{x}_{0}\right)=\overrightarrow{0}$, and 
(iv) $\vec{y}^{T}\left(\nabla^{2} f\left(\vec{x}_{0}\right)+\sum_{j=1}^{m} \alpha_{j} \nabla^{2} h_{j}\left(\vec{x}_{0}\right)+\sum_{l=1}^{p} \beta_{l} \nabla^{2} g_{l}\left(\vec{x}_{0}\right)\right) \vec{y} \geq \gamma$ for all $\vec{y} \in$ $\mathcal{R}^{n}$ satisfying $|\vec{y}|=1,\left|\nabla h_{j}\left(\vec{x}_{0}\right) \vec{y}\right|<\epsilon$ for all $j \in\{1, \ldots, m\},\left|\nabla g_{l}\left(\vec{x}_{0}\right) \vec{y}\right|<$ $\epsilon$ for all $l \in L=\left\{k: \beta_{k}>0\right\}$ and $\nabla g_{l}\left(\vec{x}_{0}\right) \vec{y}<\epsilon$ for all $l \in I\left(\vec{x}_{0}\right) \backslash L$, where $I\left(\vec{x}_{0}\right)=\left\{k: g_{k}\left(\vec{x}_{0}\right)=0\right\}$.

Then $\vec{x}_{0}$ is a strict local minimum for $f$ under the constraints of Equation (6.1).

\section{Computation of Derivatives of Real Functions}

The general question of efficient differentiation is at the core of many parts of the work on perturbation and aberration theories relevant in Physics and Engineering; for an overview, see for example [8]. In this case, derivatives of highly complicated functions have to be computed to high orders. However, even when the derivative of the function is known to exist at the given point, numerical methods fail to give an accurate value of the derivative; the error increases with the order, and for orders greater than three, the errors often become too large for the results to be practically useful.

On the other hand, while formula manipulators like Mathematica are successful in finding low-order derivatives of simple functions, they fail for high-order derivatives of very complicated functions. Moreover, they fail to find the derivatives of certain functions at given points even though the functions are differentiable at the respective points. This is generally connected to the occurrence of nondifferentiable parts that do not affect the differentiability of the end result as well as the occurrence of branch points in coding as in IF-ELSE structures.

Using Calculus on $\mathcal{R}$ and the fact that the field has infinitely small numbers represents a new method for computational differentiation that avoids the wellknown accuracy problems of numerical differentiation tools. It also avoids the often rather stringent limitations of formula manipulators that restrict the complexity of the function that can be differentiated, and the orders to which differentiation can be performed.

By a computer function, we denote any real-valued function that can be typed on a computer. The $\mathcal{R}$ numbers as well as the continuations to $\mathcal{R}$ of the intrinsic functions (and hence of all computer functions) have all been implemented for use on a computer, using the code COSY INFINITY $[\mathbf{9}, \mathbf{3 0}]$. Using the calculus on $\mathcal{R}$, we formulate a necessary and sufficient condition for the derivatives of a computer function to exist, and show how to find these derivatives whenever they exist $[40,42]$. The new technique of computing the derivatives of computer functions, which we summarize below, achieves results that combine the accuracy of formula manipulators with the speed of classical numerical methods, that is the best of both worlds.

LEMma 7.1. Let $f$ be a computer function. Then $f$ is defined at $x_{0}$ if and only if $f\left(x_{0}\right)$ can be computed on a computer.

This lemma hinges on a careful implementation of the intrinsic functions and operations, in particular in the sense that they should be executable for any floating point number in the domain of definition that produces a result within the range of allowed floating point numbers. 
LEMMA 7.2. Let $f$ be a computer function, and let $x_{0}$ be such that $f\left(x_{0}-d\right)$, $f\left(x_{0}\right)$, and $f\left(x_{0}+d\right)$ are all defined. Then $f$ is continuous at $x_{0}$ if and only if

$$
f\left(x_{0}-d\right)={ }_{0} f\left(x_{0}\right)={ }_{0} f\left(x_{0}+d\right) .
$$

If $f\left(x_{0}\right)$ and $f\left(x_{0}+d\right)$ are defined, but $f\left(x_{0}-d\right)$ is not, then $f$ is right-continuous at $x_{0}$ if and only if $f\left(x_{0}+d\right)={ }_{0} f\left(x_{0}\right)$.

Finally, if $f\left(x_{0}\right)$ and $f\left(x_{0}-d\right)$ are defined, but $f\left(x_{0}+d\right)$ is not, then $f$ is left-continuous at $x_{0}$ if and only if $f\left(x_{0}-d\right)={ }_{0} f\left(x_{0}\right)$.

THEOREM 7.3. Let $f$ be a computer function that is continuous at $x_{0}$, and let $f\left(x_{0}-d\right)$ and $f\left(x_{0}+d\right)$ be both defined. Then $f$ is differentiable at $x_{0}$ if and only if

$$
\frac{f\left(x_{0}+d\right)-f\left(x_{0}\right)}{d} \text { and } \frac{f\left(x_{0}\right)-f\left(x_{0}-d\right)}{d}
$$

are both at most finite in absolute value, and their real parts agree. In this case,

$$
\frac{f\left(x_{0}+d\right)-f\left(x_{0}\right)}{d}={ }_{0} f^{\prime}\left(x_{0}\right)={ }_{0} \frac{f\left(x_{0}\right)-f\left(x_{0}-d\right)}{d} .
$$

If $f$ is differentiable at $x_{0}$, then $f$ is twice differentiable at $x_{0}$ if and only if

$$
\frac{f\left(x_{0}+2 d\right)-2 f\left(x_{0}+d\right)+f\left(x_{0}\right)}{d^{2}} \text { and } \frac{f\left(x_{0}\right)-2 f\left(x_{0}-d\right)+f\left(x_{0}-2 d\right)}{d^{2}}
$$

are both at most finite in absolute value, and their real parts agree. In this case

$\frac{f\left(x_{0}+2 d\right)-2 f\left(x_{0}+d\right)+f\left(x_{0}\right)}{d^{2}}={ }_{0} f^{(2)}\left(x_{0}\right)={ }_{0} \frac{f\left(x_{0}\right)-2 f\left(x_{0}-d\right)+f\left(x_{0}-2 d\right)}{d^{2}}$.

In general, if $f$ is $(n-1)$ times differentiable at $x_{0}$, then $f$ is $n$ times differentiable at $x_{0}$ if and only if

$$
\frac{\sum_{j=0}^{n}(-1)^{n-j}\left(\begin{array}{c}
n \\
j
\end{array}\right) f\left(x_{0}+j d\right)}{d^{n}} \text { and } \frac{\sum_{j=0}^{n}(-1)^{j}\left(\begin{array}{c}
n \\
j
\end{array}\right) f\left(x_{0}-j d\right)}{d^{n}}
$$

are both at most finite in absolute value, and their real parts agree. In this case,

$$
\frac{\sum_{j=0}^{n}(-1)^{n-j}\left(\begin{array}{c}
n \\
j
\end{array}\right) f\left(x_{0}+j d\right)}{d^{n}}={ }_{0} f^{(n)}\left(x_{0}\right)==_{0} \frac{\sum_{j=0}^{n}(-1)^{j}\left(\begin{array}{c}
n \\
j
\end{array}\right) f\left(x_{0}-j d\right)}{d^{n}} .
$$

Since knowledge of $f\left(x_{0}-d\right)$ and $f\left(x_{0}+d\right)$ gives us all the information about a computer function $f$ in a real positive radius $\sigma$ around $x_{0}$, we have the following result which states that, from the mere knowledge of $f\left(x_{0}-d\right)$ and $f\left(x_{0}+d\right)$, we can find at once the order of differentiability of $f$ at $x_{0}$ and the accurate values of all existing derivatives.

THEOREM 7.4. Let $f$ be a computer function that is continuous at $x_{0}$. Then $f$ is $n$ times differentiable at $x_{0}$ if and only if $f\left(x_{0}-d\right)$ and $f\left(x_{0}+d\right)$ are both defined and can be written as

$$
f\left(x_{0}-d\right)={ }_{n} f\left(x_{0}\right)+\sum_{j=1}^{n}(-1)^{j} \alpha_{j} d^{j} \text { and } f\left(x_{0}+d\right)={ }_{n} f\left(x_{0}\right)+\sum_{j=1}^{n} \alpha_{j} d^{j},
$$

where the $\alpha_{j}$ 's are real numbers. Moreover, in this case $f^{(j)}\left(x_{0}\right)=j$ ! $\alpha_{j}$ for $1 \leq$ $j \leq n$. 
Now consider, as an example, the function

$$
g(x)=\frac{\sin \left(x^{3}+2 x+1\right)+\frac{3+\cos (\sin (\ln |1+x|))}{\exp \left(\tanh \left(\sinh \left(\cosh \left(\frac{\sin (\cos (\tan (\exp (x)))}{\cos (\sin (\exp (\tan (x+2)))}\right)\right)\right)\right.}}{2+\sin \left(\sinh \left(\cos \left(\tan ^{-1}\left(\ln \left(\exp (x)+x^{2}+3\right)\right)\right)\right)\right)} .
$$

Using the $\mathcal{R}$ calculus, we find $g^{(n)}(0)$ for $0 \leq n \leq 10$. These numbers are listed in table 1 ; we note that, for $0 \leq n \leq 10$, we list the CPU time needed to obtain all

TABLE 1. $g^{(n)}(0), 0 \leq n \leq 10$, computed with $\mathcal{R}$ calculus

\begin{tabular}{|c|c|c|}
\hline Order $n$ & $g^{(n)}(0)$ & CPU Time \\
\hline 0 & 1.004845319007115 & $1.820 \mathrm{msec}$ \\
\hline 1 & 0.4601438089634254 & $2.070 \mathrm{msec}$ \\
\hline 2 & -5.266097568233224 & $3.180 \mathrm{msec}$ \\
\hline 3 & -52.82163351991485 & $4.830 \mathrm{msec}$ \\
\hline 4 & -108.4682847837855 & $7.700 \mathrm{msec}$ \\
\hline 5 & 16451.44286410806 & $11.640 \mathrm{msec}$ \\
\hline 6 & 541334.9970224757 & $18.050 \mathrm{msec}$ \\
\hline 7 & 7948641.189364974 & $26.590 \mathrm{msec}$ \\
\hline 8 & -144969388.2104904 & $37.860 \mathrm{msec}$ \\
\hline 9 & -15395959663.01733 & $52.470 \mathrm{msec}$ \\
\hline 10 & -618406836695.3634 & $72.330 \mathrm{msec}$ \\
\hline
\end{tabular}

derivatives of $g$ at 0 up to order $n$ and not just $g^{(n)}(0)$. For comparison purposes, we give in table 2 the function value and the first six derivatives computed with

TABLE 2. $g^{(n)}(0), 0 \leq n \leq 6$, computed with Mathematica

\begin{tabular}{|c|c|c|}
\hline Order $n$ & $g^{(n)}(0)$ & CPU Time \\
\hline 0 & 1.004845319007116 & $0.11 \mathrm{sec}$ \\
\hline 1 & 0.4601438089634254 & $0.17 \mathrm{sec}$ \\
\hline 2 & -5.266097568233221 & $0.47 \mathrm{sec}$ \\
\hline 3 & -52.82163351991483 & $2.57 \mathrm{sec}$ \\
\hline 4 & -108.4682847837854 & $14.74 \mathrm{sec}$ \\
\hline 5 & 16451.44286410805 & $77.50 \mathrm{sec}$ \\
\hline 6 & 541334.9970224752 & $693.65 \mathrm{sec}$ \\
\hline
\end{tabular}

Mathematica. Note that the respective values listed in tables 1 and 2 agree. However, Mathematica used much more CPU time to compute the first six derivatives, and it failed to find the seventh derivative as it ran out of memory. We also list in table 3 the first ten derivatives of $g$ at 0 computed numerically using the numerical differentiation formulas

$$
g^{(n)}(0)=(\Delta x)^{-n}\left(\sum_{j=0}^{n}(-1)^{n-j}\left(\begin{array}{c}
n \\
j
\end{array}\right) g(j \Delta x)\right), \Delta x=10^{-16 /(n+1)},
$$

for $1 \leq n \leq 10$, together with the corresponding relative errors obtained by comparing the numerical values with the respective exact values computed using $\mathcal{R}$ calculus. 
TABLE 3. $g^{(n)}(0), 1 \leq n \leq 10$, computed numerically

\begin{tabular}{|c|c|c|}
\hline Order $n$ & $g^{(n)}(0)$ & Relative Error \\
\hline 1 & 0.4601437841866840 & $54 \times 10^{-9}$ \\
\hline 2 & -5.266346392944456 & $47 \times 10^{-6}$ \\
\hline 3 & -52.83767867680922 & $30 \times 10^{-5}$ \\
\hline 4 & -87.27214664649106 & 0.20 \\
\hline 5 & 19478.29555909866 & 0.18 \\
\hline 6 & 633008.9156614641 & 0.17 \\
\hline 7 & -12378052.73279768 & 2.6 \\
\hline 8 & -1282816703.632099 & 7.8 \\
\hline 9 & 83617811421.48561 & 6.4 \\
\hline 10 & 91619495958355.24 & 149 \\
\hline
\end{tabular}

On the other hand, formula manipulators fail to find the derivatives of certain functions at given points even though the functions are differentiable at the respective points. For example, the functions

$$
g_{1}(x)=|x|^{5 / 2} \cdot g(x) \text { and } g_{2}(x)= \begin{cases}\frac{1-\exp \left(-x^{2}\right)}{x} \cdot g(x) & \text { if } x \neq 0 \\ 0 & \text { if } x=0\end{cases}
$$

where $g(x)$ is the function given in Equation (7.1), are both differentiable at 0; but the attempt to compute their derivatives using formula manipulators fails. This is not specific to $g_{1}$ and $g_{2}$, and is generally connected to the occurrence of nondifferentiable parts that do not affect the differentiability of the end result, of which case $g_{1}$ is an example, as well as the occurrence of branch points in coding as in IF-ELSE structures, of which case $g_{2}$ is an example.

\section{Existence and Uniqueness of Solutions of Ordinary Differential Equations}

In [10], we consider differential equations over $\mathcal{R}$ with a right hand side that is infinitely often derivate differentiable. We show that such an ODE admits solutions that are themselves infinitely often derivate differentiable. To this end, we develop a theory of multivariate infinitely often derivate differentiable functions and show that they can be locally represented as Taylor series. We then re-phrase the ODE problem as a fixed point problem of a Picard operator in the common way. After various transformations and utilizing well-known existence and uniqueness properties of ODEs over $\mathbb{R}$, the problem is transformed to a fixed point problem with an infinitely small contraction factor. We show that the sequence of functions obtained by iteration converges uniformly in the order topology, that the resulting limit is itself infinitely often derivate differentiable, and that this limit indeed solves the ODE. It is then shown that while there are other solutions with lesser smoothness requirements, the solution so obtained is unique among all the infinitely often derivate differentiable functions. 


\section{Current and Future Research}

Several research projects are currently under way. Building on the existing knowledge of the field, which has been summarized in the sections above, we are working on developing a multivariate calculus theory on $\mathcal{R}^{n}$ as well as on completing the study of power series and $\mathcal{R}$-analytic functions by proving the Extreme Value Theorem and the Mean Value Theorem. We plan also to do more analysis on $\mathcal{C}$ and look into more potential applications of the implementation of the $\mathcal{R}$ numbers on a computer. Moreover, in collaboration with Jose Aguayo and Miguel Nova, we are looking into the possibility of developing a non-Archimedean Hilbert Space theory by considering the space $c_{0}$ of null sequences of elements of $\mathcal{R}$, equipped with the non-Archimedean inner product $\left\langle\left(x_{n}\right),\left(y_{n}\right)\right\rangle:=\sum_{n=1}^{\infty} x_{n} y_{n}$; this may lead to some useful applications in Physics, particularly in Quantum Mechanics.

\section{Acknowledgments}

The authors wish to thank the kind referee for the useful comments and suggestions which helped improve the quality of the paper.

\section{References}

[1] N. L. Alling. Foundations of Analysis over Surreal Number Fields. North Holland, 1987.

[2] S. Basu, R. Pollack, and M. Roy. Algorithms in Real Algebraic Geometry. Springer, 2003.

[3] M. Berz. Analysis on a nonarchimedean extension of the real numbers. Lecture Notes, 1992 and 1995 Mathematics Summer Graduate Schools of the German National Merit Foundation. MSUCL-933, Department of Physics, Michigan State University, 1994.

[4] M. Berz. Calculus and numerics on Levi-Civita fields. In M. Berz, C. Bischof, G. Corliss, and A. Griewank, editors, Computational Differentiation: Techniques, Applications, and Tools, pages 19-35, Philadelphia, 1996. SIAM.

[5] M. Berz. Analytical and computational methods for the Levi-Civita fields. In Proc. Sixth International Conference on Nonarchimedean Analysis, pages 21-34, New York, NY, 2000. Marcel Dekker.

[6] M. Berz. Nonarchimedean analysis and rigorous computation. International Journal of Applied Mathematics, 2:889-930, 2000.

[7] M. Berz. Cauchy theory on Levi-Civita fields. Contemporary Mathematics, 319:39-52, 2003.

[8] M. Berz, C. Bischof, A. Griewank, G. Corliss, and Eds. Computational Differentiation: Techniques, Applications, and Tools. SIAM, Philadelphia, 1996.

[9] M. Berz, G. Hoffstätter, W. Wan, K. Shamseddine, and K. Makino. COSY INFINITY and its applications to nonlinear dynamics. In M. Berz, C. Bischof, G. Corliss, and A. Griewank, editors, Computational Differentiation: Techniques, Applications, and Tools, pages 363-367, Philadelphia, 1996. SIAM.

[10] M. Berz and K. Shamseddine. Existence and uniqueness of solutions of differential equations on the Levi-Civita field. in preparation.

[11] J. H. Conway. On Numbers and Games. North Holland, 1976.

[12] M. Davis. Applied Nonstandard Analysis. John Wiley and Sons, 1977.

[13] H.-D. Ebbinghaus et al. Zahlen. Springer, 1992.

[14] Anthony V. Fiacco and Garth P. McCormick. Nonlinear Programming; Sequential Unconstrained Minimization Techniques. SIAM, Philadelphia, 1990.

[15] L. Fuchs. Partially Ordered Algebraic Systems. Pergamon Press, Addison Wesley, 1963.

[16] H. Gonshor. An Introduction to the Theory of Surreal Numbers. Cambrindge University Press, 1986.

[17] H. Hahn. Über die nichtarchimedischen Größensysteme. Sitzungsbericht der Wiener Akademie der Wissenschaften Abt. 2a, 117:601-655, 1907.

[18] E. Hewitt and K. Stromberg. Real and Abstract Analysis. Springer, 1969.

[19] D. E. Knuth. Surreal Numbers: How two ex-students turned on to pure mathematics and found total happiness. Addison-Wesley, 1974. 
[20] W. Krull. Allgemeine Bewertungstheorie. J. Reine Angew. Math., 167:160-196, 1932.

[21] D. Laugwitz. Eine Einführung der Delta-Funktionen. Sitzungsberichte der Bayerischen Akademie der Wissenschaften, 4:41, 1959.

[22] D. Laugwitz. Anwendungen unendlich kleiner Zahlen I. Journal für die reine und angewandte Mathematik, 207:53-60, 1961.

[23] D. Laugwitz. Anwendungen unendlich kleiner Zahlen II. Journal für die reine und angewandte Mathematik, 208:22-34, 1961.

[24] D. Laugwitz. Ein Weg zur Nonstandard-Analysis. Jahresberichte der Deutschen Mathematischen Vereinigung, 75:66-93, 1973.

[25] D. Laugwitz. Tullio Levi-Civita's work on nonarchimedean structures (with an Appendix: Properties of Levi-Civita fields). In Atti Dei Convegni Lincei 8: Convegno Internazionale Celebrativo Del Centenario Della Nascita De Tullio Levi-Civita, Academia Nazionale dei Lincei, Roma, 1975.

[26] T. Levi-Civita. Sugli infiniti ed infinitesimi attuali quali elementi analitici. Atti Ist. Veneto di Sc., Lett. ed Art., 7a, 4:1765, 1892.

[27] T. Levi-Civita. Sui numeri transfiniti. Rend. Acc. Lincei, 5a, 7:91,113, 1898.

[28] A. H. Lightstone and A. Robinson. Nonarchimedean Fields and Asymptotic Expansions. North Holland, New York, 1975.

[29] David G. Luenberger. Linear and Nonlinear Programming. Addison-Wesley, Reading, Massachusetts, 2nd edition, 1984.

[30] K. Makino and M. Berz. COSY INFINITY version 9. Nuclear Instruments and Methods, A558:346-350, 2005.

[31] L. Neder. Modell einer Leibnizschen Differentialrechnung mit aktual unendlich kleinen Größen. Mathematische Annalen, 118:718-732, 1941-1943.

[32] S. Priess-Crampe. Angeordnete Strukturen: Gruppen, Körper, projektive Ebenen. Springer, Berlin, 1983.

[33] P. Ribenboim. Fields: Algebraically Closed and Others. Manuscripta Mathematica, 75:115150, 1992.

[34] A. Robinson. Non-standard analysis. In Proceedings Royal Academy Amsterdam, Series A, volume 64, page 432, 1961.

[35] A. Robinson. Non-Standard Analysis. North-Holland, 1974.

[36] W. Rudin. Real and Complex Analysis. McGraw Hill, 1987.

[37] W. H. Schikhof. Ultrametric Calculus: An Introduction to p-Adic Analysis. Cambridge University Press, 1985.

[38] C. Schmieden and D. Laugwitz. Eine Erweiterung der Infinitesimalrechnung. Mathematische Zeitschrift, 69:1-39, 1958.

[39] K. Shamseddine. New Elements of Analysis on the Levi-Civita Field. PhD thesis, Michigan State University, East Lansing, Michigan, USA, 1999. also Michigan State University report MSUCL-1147.

[40] K. Shamseddine and M. Berz. Exception handling in derivative computation with nonArchimedean calculus. In M. Berz, C. Bischof, G. Corliss, and A. Griewank, editors, Computational Differentiation: Techniques, Applications, and Tools, pages 37-51, Philadelphia, 1996. SIAM.

[41] K. Shamseddine and M. Berz. Convergence on the Levi-Civita field and study of power series. In Proc. Sixth International Conference on Nonarchimedean Analysis, pages 283-299, New York, NY, 2000. Marcel Dekker.

[42] K. Shamseddine and M. Berz. The differential algebraic structure of the Levi-Civita field and applications. International Journal of Applied Mathematics, 3:449-465, 2000.

[43] K. Shamseddine and M. Berz. Intermediate values and inverse functions on non-Archimedean fields. International Journal of Mathematics and Mathematical Sciences, 30:165-176, 2002.

[44] K. Shamseddine and M. Berz. Measure theory and integration on the Levi-Civita field. Contemporary Mathematics, 319:369-387, 2003.

[45] K. Shamseddine and M. Berz. Analytical properties of power series on Levi-Civita fields. Annales Mathématiques Blaise Pascal, 12(2):309-329, 2005.

[46] K. Shamseddine and M. Berz. Generalized power series on a non-Archimedean field. Indagationes Mathematicae, 17(3):457-477, 2006.

[47] K. Shamseddine and M. Berz. Intermediate value theorem for analytic functions on a LeviCivita field. Bulletin of the Belgian Mathematical Society-Simon Stevin, 14:1001-1015, 2007. 
[48] K. Shamseddine and V. Zeidan. One-dimensional optimization on non-Archimedean fields. Journal of Nonlinear and Convex Analysis, 2:351-361, 2001.

[49] K. Shamseddine and V. Zeidan. Constrained second order optimization on non-Archimedean fields. Indagationes Mathematicae, 14:81-101, 2003.

[50] K. Stromberg. An Introduction to Classical Real Analysis. Wadsworth, 1981.

[51] K. D. Stroyan and W. A. J. Luxemburg. Introduction to the Theory of Infinitesimals. Academic Press, 1976

Department of Physics and Astronomy, University of Manitoba, Winnipeg, ManiTOBA R3T 2N2, CANADA

E-mail address: khodr@physics.umanitoba.ca

Department of Physics and Astronomy, Michigan State University, East Lansing, MI 48824, USA

E-mail address: martin.berz@gmx.de 